\title{
A RADON-NIKODYM THEOREM FOR STONE ALGEBRA VALUED MEASURES
}

\author{
BY \\ J. D. MAITLAND WRIGHT
}

Introduction. Let $C(S)$ be the ring of continuous real valued functions on a compact Hausdorff space $S$. Stone [5] shows that each bounded subset of $C(S)$ has a least upper bound (in $C(S)$ ) if and only if the closure of each open subset of $S$ is open; in this event we call $C(S)$ a Stone algebra. Throughout this paper $C(S)$ is a Stone algebra.

It is convenient to adjoin an object $+\infty$, not in $C(S)$, and extend the partial ordering of $C(S)$ to $C(S) \cup\{+\infty\}$ in the obvious way. When $\left\{a_{n}: n=1,2, \ldots\right\}$ is an unbounded set in $C(S)$ we define $\bigvee_{n=1}^{\infty} a_{n}$ to be $+\infty$.

Following [6] we define a $C(S)$-valued measure on a measurable space $(X, \mathscr{B})$ to be a map $m: \mathscr{B} \rightarrow C(S) \cup\{+\infty\}$ such that $m E \geqq 0$ for each $E$ and, if $\left\{E_{n}\right\}$ $n=1,2, \ldots$ is a pairwise disjoint sequence of sets in $\mathscr{B}$ then

$$
m \bigcup_{n=1}^{\infty} E_{n}=\bigvee_{n=1}^{\infty} \sum_{r=1}^{n} m E_{r} .
$$

In the special case where $C(S)$ is identifiable with the self-adjoint part of a commutative von Neumann algebra the latter condition is equivalent to

$$
m E=\lim \sum_{r=1}^{n} m E_{r}
$$

where the limit is taken in the weak operator topology. But Floyd [1] gives an example of a Stone algebra such that there is no Hausdorff vector topology in which each bounded monotone increasing sequence converges to its least upper bound. This pathology causes some difficulty.

In [6] a theory of Stone algebra valued measures and integrals was constructed and in [7] this was applied to give a spectral theorem for normal operators on a Kaplansky-Hilbert module. It is not necessary to have read these papers to understand the work here although we shall use some of the results obtained in [6].

The goal of this paper is an analogue for Stone algebra valued measures of the classical Radon-Nikodym Theorem. In a later publication I intend to discuss applications of the results of this paper to averaging operators and to Boolean algebras.

The following analogue of the Radon-Nikodym.Theorem might be conjectured.

Received by the editors November 3, 1967 and, in revised form, June 17, 1968. 
Let $l$ and $m$ be finite $C(S)$-valued measures on a measurable space $(X, \mathscr{B})$ and suppose that $m E=0$ implies $l E=0$. Then there exists a $\mathscr{B}$-measurable function $f: X \rightarrow \boldsymbol{R}$ such that $l E=\int_{E} f d m$ for each $E \in \mathscr{B}$. It is easy to show that this is false. Let $X=S=\{a, b\}$, a two-point space, then $C(S)$ can be identified with the space of all ordered pairs of real numbers. Let $\mathscr{B}$ be the collection of all subsets of $X$. Define measures $l$ and $m$ by

$$
l\{a\}=m\{b\}=(1,0) \quad \text { and } \quad l\{b\}=m\{a\}=(0,1) .
$$

Assume $f$ exists and take $E=\{a\}$. Then

$$
(1,0)=l\{a\}=\int_{\{a\}} f d m=f(a)(0,1)
$$

so that $(1,0)$ is a scalar multiple of $(0,1)$ which is impossible.

In $\$ 4$ a generalization of the Radon-Nikodym Theorem is obtained. In the example above the measures are "twisted" relative to each other. We restrict ourselves to modular measures and are then able to define absolute continuity in such a way as to exclude "twisting".

Modular measures are defined in $\S 2$. When a $C(S)$-valued measure is modular its $L^{p}$-spaces form modules over $C(S)$ and integration with respect to the measure is a module homomorphism of $L^{1}$ into $C(S)$.

When $C(S)$ satisfies the countable chain condition and the measure is modular then $L^{2}$ is a Kaplansky-Hilbert module. This gives new examples of KaplanskyHilbert modules. More important for our purpose is that it enables us to use Kaplansky's powerful results.

It is now of interest to know when a Stone algebra satisfies the countable chain condition. If $S$ is the Boolean space of a complete Boolean algebra satisfying the countable chain condition then $C(S)$ also satisfies the countable chain condition. It is well known that when $\mu$ is a finite real valued measure on a space $(X, \mathscr{B})$ then $L^{\infty}(X, \mathscr{B}, \mu)$ satisfies the countable chain condition. We show that, when $m$ is a finite $C(S)$-valued measure, if $C(S)$ satisfies the countable chain condition then so also does $L^{\infty}(X, \mathscr{B}, m)$ and thus $L^{\infty}(X, \mathscr{B}, m)$ is a Stone algebra.

For the Radon-Nikodym Theorem to hold and for $L^{2}$-spaces to be KaplanskyHilbert modules it is not essential that the modular measures take their values in a Stone algebra satisfying the countable chain condition but this is a simple and convenient condition for applications.

1. $L^{p}$-spaces. Throughout this paper, unless we state otherwise, $X$ is a nonempty set and $\mathscr{B}$ is a $\sigma$-algebra of subsets of $X ; m: \mathscr{B} \rightarrow C(S)$ is a positive $C(S)$ valued measure which is $\sigma$-additive in the sense defined in the introduction; $\mathscr{L}^{1}(X, m)$ is the set of real valued $m$-integrable functions on $X$; for $1 \leqq p<\infty$, $\mathscr{L}^{p}(X, m)$ is the set of $\mathscr{B}$-measurable functions $f$ such that $|f|^{p} \in \mathscr{L}^{1}(X, m)$; $\mathscr{L}^{\infty}(X, m)$ is the set of $\mathscr{B}$-measurable functions such that for each $f \in \mathscr{L}^{\infty}(X, m)$ there can be found $F \in \mathscr{B}$ with $m F=0$ and $f$ bounded on $X-F$. 
We call $(X, \mathscr{B}, m, C(S))$ a complete $C(S)$-measure space if $m E=0$ and $N \subset E$ implies $N \in \mathscr{B}$.

Lemma 1.1. Let $N \in \mathscr{N}$ iff $N \subset E$ for some $E \in \mathscr{B}$ such that $m E=0$. Let $\mathscr{B} *$ be the collection of all sets of the form $B \cup N$ where $B \in \mathscr{B}$ and $N \in \mathscr{N}$. Then $\mathscr{B} *$ is a o-algebra of subsets of $X$ and there is a unique measure $m^{*}: \mathscr{B}^{*} \rightarrow C(S) \cup\{+\infty\}$ such that $m^{*} B=m B$ for each $B \in \mathscr{B}$. Moreover $\left(X, \mathscr{B}^{*}, m^{*}, C(S)\right)$ is a complete $C(S)$-measure space and if $f^{*}$ is $\mathscr{B}^{*}$-measurable there can be found a $\mathscr{B}$-measurable function $f$ such that $m^{*}\left\{x \in X: f^{*}(x) \neq f(x)\right\}=0$.

The first part of the lemma is straightforward. Let $f^{*}$ be a positive $\mathscr{B}^{*}$-measurable function and $\left\{f_{n}^{*}\right\}, n=1,2, \ldots$, a monotone increasing sequence of $\mathscr{B}^{*}$-simple functions such that $f^{*}(x)=\sup f_{n}^{*}(x)$ for each $x$. For each $n$ we can find a $\mathscr{B}$-simple function $f_{n}$ such that $f_{n} \leqq f_{n}^{*}$ and $m^{*}\left\{x: f_{n}(x)<f_{n}^{*}(x)\right\}=0$. Let $f=\sup f_{n}$ then $f \leqq f^{*}$ and

$$
\begin{aligned}
\left\{x: f^{*}(x) \neq f(x)\right\} & =\bigcup_{n=1}^{\infty}\left\{x: f(x)<f_{n}^{*}(x)\right\} \\
& \subset \bigcup_{n=1}^{\infty}\left\{x: f_{n}(x)<f_{n}^{*}(x)\right\} .
\end{aligned}
$$

So $m^{*}\left\{x: f(x) \neq f^{*}(x)\right\}=0$ and the result follows.

LeMma 1.2. For $1 \leqq p \leqq \infty, \mathscr{L}^{p}(X, m)$ is a vector lattice.

This is a trivial consequence of the definition of $\mathscr{L}^{p}(X, m)$ and the inequality, valid for $1 \leqq p<\infty$,

$$
|f+g|^{p} \leqq(|f|+|g|)^{p} \leqq 2^{p}\left(|f|^{p}+|g|^{p}\right) .
$$

When $f$ and $g$ are $\mathscr{B}$-measurable functions $f$ is said to be equivalent to $g, f \sim g$, if $m\{x: f(x) \neq g(x)\}=0$. It is easy to verify that this does define an equivalence relation; that $f \sim g$ iff $f-g \sim 0$; and that the collection of functions equivalent to 0 is a subspace of $\mathscr{L}^{p}(X, m)$. We define $L^{p}(X, m)$ to be the quotient of $\mathscr{L}^{p}(X, m)$ by this equivalence relation, then $L^{p}(X, m)$ is a vector lattice with respect to the induced vector operations and partial ordering. Although $\mathscr{L}^{p}(X, m)$ may be properly contained in $\mathscr{L}^{p}\left(X, m^{*}\right)$ it follows from Lemma 1.1 that $L^{p}(X, m)=L^{p}\left(X, m^{*}\right)$.

For the rest of this paper, unless we specify otherwise, $\mathscr{L}^{p}(X)$ is $\mathscr{L}^{p}\left(X, m^{*}\right)$ and $L^{p}(X)$ is $L^{p}(X, m)=L^{p}\left(X, m^{*}\right)$.

When $f$ is a $\mathscr{B}^{*}$-measurable function we define $[f]$ to be the set of all $\mathscr{B}^{*}$-measurable functions equivalent to $f$. This notation will sometimes be misused by writing $f$ for $[f]$ and, when $[f]=\xi$, by writing $\xi$ for $f$.

As in classical measure theory the essential supremum of the modulus of a function is defined by

$$
\text { ess sup }|f|=\inf \left\{\lambda \in \boldsymbol{R}^{+}: m\{x:|f(x)|>\lambda\}=0\right\} \text {. }
$$


A measurable function on $X$ is in $\mathscr{L}^{\infty}(X, m)$ if and only if its modulus has a finite essential supremum. The essential supremum of the modulus of a function defines a seminorm on $\mathscr{L}^{\infty}(X, m)$ which induces a norm on $L^{\infty}(X, m)$.

Lemma 1.3. When $L^{\infty}(X)$ is given the essential supremum norm and the natural multiplication it is a Banach algebra and is isometrically isomorphic to the algebra of all real valued continuous functions on some compact Hausdorff space.

Let $B^{\infty}(X)$ be the algebra of bounded real valued $\mathscr{B}$-measurable functions on $X$ equipped with the supremum norm and let $\mathscr{M}$ be the subspace of $B^{\infty}(X)$ consisting of functions vanishing outside a set of zero $m$-measure. Then $B^{\infty}(X)$ is the selfadjoint part of a commutative $C^{*}$-algebra and so isometrically isomorphic to $C(K)$ for some compact Hausdorff $K$. Further $\mathscr{M}$ is a closed ideal and $L^{\infty}(X)$ can be identified in a natural way with $B^{\infty}(X) / \mathscr{M}$. The result follows by verifying that this identification induces the same norm on $L^{\infty}(X)$ as the essential supremum norm.

LEMMA 1.4. Let $f \in \mathscr{L}^{p}(X, m)$ and $g \in \mathscr{L}^{q}(X, m)$ where $1<p<\infty$ and $1 / p+1 / q=1$ then $f g \in \mathscr{L}^{1}(X, m)$ and $|f g| \leqq p^{-1}|f|^{p}+q^{-1}|g|^{q}$.

When $0<\lambda<1$ and $\alpha$ and $\beta$ are positive real numbers the inequality $\alpha^{\lambda} \beta^{1-\lambda}$ $\leqq \lambda \alpha+(1-\lambda) \beta$ is well known. Put $\alpha=|f(x)|^{p}, \beta=|g(x)|^{q}$ and $\lambda=1 / p$ then the required inequality follows. Thus $f g$ is a measurable function dominated by a function in $\mathscr{L}^{1}(X, m)$ so $f g$ is in $\mathscr{L}^{1}(X, m)$.

We observe that if $f \in \mathscr{L}^{\infty}(X)$ and $g \in \mathscr{L}^{p}(X)$ then $f g \in \mathscr{L}^{p}(X)$. For any measurable $f$ and $g$ we define $[f][g]$ to be $[f g]$.

Lemma 1.5. Let $1<p<\infty$ and $1 / p+1 / q=1$. If $f \in \mathscr{L}^{p}(X, m)$ and $g \in \mathscr{L}^{q}(X, m)$ then

$$
\int_{X}|f g| d m \leqq\left(\int_{X}|f|^{p} d m\right)^{1 / p}\left(\int_{X}|g|^{q} d m\right)^{1 / q} .
$$

Let $Y$ be a subset of $X$ with $m Y<+\infty$ and let $a$ and $b$ be positive elements of $L^{\infty}(Y, m)$. By Lemma 1.3 we may identify $L^{\infty}(Y, m)$ with $C(E)$ for some compact Hausdorff space $E$. Misusing our notation, we define $T: C(E) \rightarrow C(S)$ by $T h$ $=\int_{Y} h d m$.

For each $s \in S$ the map $h \rightarrow(T h)(s)$ is a positive linear functional on $C(E)$. Let $\mu_{s}$ be the unique (real valued) Baire measure on $E$ such that

$$
\int_{E} h d \mu_{s}=(T h)(s) \text { for each } h \in C(E) .
$$

By the classical Hölder inequality,

$$
\int_{E} a b d \mu_{s} \leqq\left(\int_{E} a^{p} d \mu_{s}\right)^{1 / p}\left(\int_{E} b^{q} d \mu_{s}\right)^{1 / q} .
$$


So

$$
(T a b)(s) \leqq\left(T a^{p}\right)^{1 / p}(s)\left(T b^{q}\right)^{1 / q}(s)
$$

Thus

$$
\int_{Y} a b d m \leqq\left(\int_{Y} a^{p} d m\right)^{1 / p}\left(\int_{Y} b^{q}\right)^{1 / q}
$$

Let $f \in \mathscr{L}^{p}(X, m)$ and $g \in \mathscr{L}^{q}(X, m)$. Then we can find monotone increasing sequences of positive simple functions $\left\{f_{n}\right\}, n=1,2, \ldots$ and $\left\{g_{n}\right\}, n=1,2, \ldots$ such that $f_{n} \rightarrow|f|$ and $g_{n} \rightarrow|g|$. For each $n, f_{n}$ and $g_{n}$ vanish outside a set of finite $m$-measure and are bounded functions. So

$$
\begin{aligned}
\int_{X} f_{n} g_{n} d m & \leqq\left(\int_{X} f_{n}^{p} d m\right)^{1 / p}\left(\int_{X} g_{n}^{q} d m\right)^{1 / q} \\
& \leqq\left(\int_{X}|f|^{p} d m\right)^{1 / p}\left(\int_{X}|g|^{q} d m\right)^{1 / q} .
\end{aligned}
$$

By Proposition 3.3 of [6]

$$
\bigvee_{n=1}^{\infty} \int_{X} f_{n} g_{n} d m=\int_{X}|f g| d m
$$

This completes the proof.

We introduce the notation $\phi([f])=\int_{X} f d m^{*}$ for $f \in \mathscr{L}^{1}\left(X, m^{*}\right)$.

LEMMA 1.6. If $1 \leqq p<\infty$ and $f, g$ are in $\mathscr{L}^{p}(X)$, then

$$
\phi\left(|f+g|^{p}\right)^{1 / p} \leqq \phi\left(|f|^{p}\right)^{1 / p}+\phi\left(|g|^{p}\right)^{1 / p} .
$$

The inequality is trivial when $p=1$. Suppose $1<p$ and let $q=p /(p-1)$ so that $1 / p+1 / q=1$. Since $|f+g|^{p-1}$ is in $\mathscr{L}^{q}(X)$ we have by Lemma 1.5

$$
\begin{aligned}
\phi\left(|f+g|^{p-1}|f|\right) & \leqq \phi\left(|f+g|^{(p-1) q}\right)^{1 / q} \phi\left(|f|^{p}\right)^{1 / p} \\
& =\phi\left(|f+g|^{p}\right)^{1-1 / p} \phi\left(|f|^{p}\right)^{1 / p} .
\end{aligned}
$$

But

$$
\phi\left(|f+g|^{p}\right) \leqq \phi\left(|f+g|^{p-1}|f|\right)+\phi\left(|f+g|^{p-1}|g|\right) .
$$

So

$$
\phi\left(|f+g|^{p}\right) \leqq \phi\left(|f+g|^{p}\right)^{1-1 / p}\left\{\phi\left(|f|^{p}\right)^{1 / p}+\phi\left(|g|^{p}\right)^{1 / p}\right\}
$$

and the result follows.

It is now clear that for $1 \leqq p<\infty$ we can define a seminorm \|\|$_{p}$ on $\mathscr{L}^{p}(X)$ by defining $\|f\|_{p}$ to be the norm of $\phi\left(|f|^{p}\right)^{1 / p}$ as an element of $C(S)$. Then \|\|$_{p}$ induces a norm on $L^{p}(X)$ which we will also denote by \|\|$_{p}$.

Order limits and order sums were defined in [6] as follows. If $\left\{a_{r}\right\}, r=1,2, \ldots$, is a $C(S)$-valued sequence and

$$
\bigvee_{n=1}^{\infty} \bigwedge_{r=n}^{\infty} a_{r}=\bigwedge_{n=1}^{\infty} \bigvee_{r=n}^{\infty} a_{r}
$$


then this common value is $\operatorname{LIM} a_{r}$. Further $\sum_{1} \hat{a} a_{r}$ is the order limit of the partial sums $\left\{\sum_{1}^{n} a_{r}\right\}, n=1,2, \ldots$

Proposition 1.1. Let $f_{n} \in \mathscr{L}^{p}(X)$ for $n=1,2, \ldots$ where $1 \leqq p<\infty$ and suppose $\sum_{1} \hat{\phi}\left(\left|f_{n}\right|^{p}\right)^{1 / p}<\infty$. Then there is an $f \in \mathscr{L}^{p}(X)$ such that

$$
\operatorname{LIM}_{n} \phi\left(\left|f-\sum_{1}^{n} f_{r}\right|^{p}\right)^{1 / p}=0 .
$$

Let $g_{n}(x)=\sum_{1}^{n}\left|f_{r}(x)\right|$ for each $x \in X$. Then, by repeated applications of Lemma 1.6,

$$
\phi\left(g_{n}^{p}\right)^{1 / p} \leqq \sum_{1}^{n} \phi\left(\left|f_{r}\right|^{p}\right)^{1 / p}
$$

By hypothesis there exists $b \in C(S)$ such that

$$
\bigvee_{n=1}^{\infty} \sum_{1}^{n} \phi\left(\left|f_{r}\right|^{p}\right)^{1 / p}=b .
$$

So $\phi\left(g_{n}^{p}\right) \leqq b^{p}$ for all $n$.

Let $g(x)=\sup g_{n}(x)$ for each $x \in X$. By Proposition 3.3 of [6]

$$
\phi\left(g^{p}\right)=\bigvee_{n=1}^{\infty} \phi\left(g_{p}^{n}\right) \leqq b^{p}
$$

Let $E=\{x: g(x)=+\infty\}$ then $m^{*} E=0$. For $x \notin E, \sum_{1}^{\infty}\left|f_{r}(x)\right|$ is convergent. Let $f(x)=\sum_{1}^{\infty} f_{r}(x)$ for $x \notin E$ and $f(x)=0$ for $x \in E$. So $|f|^{p} \leqq g^{p}$ and thus $f \in \mathscr{L}^{p}(X)$.

We have $\left\{\left|f-\sum_{1}^{n} f_{r}\right|^{p}\right\}, n=1,2, \ldots$, converges pointwise to zero, except in $E$, and each term is dominated by $(|f|+|g|)^{p} \leqq 2^{p}|g|^{p}$. By Proposition 3.5 of [6] it follows that $\operatorname{LIM}_{n} \phi\left(\left|f-\sum_{1}^{n} f_{r}\right|^{p}\right)$ exists and is zero. It follows from Lemma 1.1 of [6] that $\operatorname{LIM}_{n} \phi\left(\left|f-\sum_{1}^{n} f_{r}\right|^{p}\right)^{1 / p}=0$.

Proposition 1.2. If $1 \leqq p<\infty$ then $L^{p}(X)$ is a Banach space with respect to \|\|$_{p}$.

Let $\sum_{r=1}^{\infty} f_{r}$ be such that $\sum_{1}^{\infty}\left\|f_{r}\right\|_{p}<\infty$. Then it follows from Proposition 1.1 that there exists $f \in \mathscr{L}^{p}(X)$ such that

$$
\operatorname{LIM} \phi\left(\left|f-\sum_{1}^{n} f_{r}\right|^{p}\right)^{1 / p}=0
$$

It follows from the proof of Proposition 1.1 that we can suppose

$$
f(x)=\lim _{n} \sum_{1}^{n} f_{r}(x)
$$

and that $\sum_{1}^{\infty}\left|f_{r}(x)\right|$ is convergent almost everywhere. 
Choose a positive real number $\varepsilon$. There exists $N=N(\varepsilon)$ such that $\sum_{r=N}^{\infty}\left\|f_{r}\right\|_{p}<\varepsilon$. For any $l>m>N$ we have

So

$$
\phi\left(\left|\sum_{r=m}^{l} f_{r}\right|^{p}\right)^{1 / p} \leqq \sum_{r=m}^{l} \phi\left(\left|f_{r}\right|^{p}\right)^{1 / p} .
$$

$$
\left\|\sum_{r=m}^{l} f_{r}\right\|_{p} \leqq \sum_{r=m}^{l}\left\|f_{r}\right\|_{p} \leqq \varepsilon .
$$

By Proposition 3.4 of [6]

So

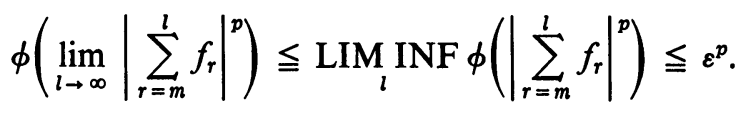

$$
\phi\left(\left|f-\sum_{1}^{m-1} f_{r}\right|^{p}\right) \leqq \varepsilon^{p} .
$$

Thus $\left\|f-\sum_{1}^{m-1} f_{r}\right\|_{p} \leqq \varepsilon$ whenever $m>N=N(\varepsilon)$. Thus $L^{p}(X)$ is a Banach space.

2. Modular measures. To avoid some rather dull pathology we shall assume for the rest of this paper that each set of infinite measure contains a set of finite nonzero measure, that is, if $A \in \mathscr{B}$ such that $m A=+\infty$ then there exists $F \subset A$ such that $0<m F<m A$.

LEMMA 2.1. Let $g \in \mathscr{L}^{\infty}(X, \mathscr{B}, m)$ such that $\int_{X} g f d m=0$ for each $f \in \mathscr{L}^{1}(X, \mathscr{B}, m)$. Then $[g]=0$.

Let $E=\{x \in X: g(x)>0\}$. Then $g\left(2 \chi_{E}-1\right)=g^{+}+g^{-}=|g|$. For each $f \in \mathscr{L}^{1}(X, m)$ the function $\left(2 \chi_{E}-1\right) f \in \mathscr{L}^{1}(X, m)$ and so

$$
\int_{X}|g| f d m=\int_{X} g\left(2 \chi_{E}-1\right) f d m=0 .
$$

Let $A_{n}=\{x \in X:|g|(x) \geqq 1 / n\}$. If $m A_{n} \neq 0$ then there exists a set $F \subset A_{n}$ such that $0<m F<+\infty$. Then $\int_{X}|g| \chi_{F} d m=0$ and so $m F / n=0$, that is, $m F=0$. Therefore the assumption that $m A_{n} \neq 0$ is false and so $[g]=0$.

LEMMA 2.2. If there exists a function $\pi: C(S) \rightarrow L^{\infty}(X, m)$ such that

$$
\int_{X} \pi(a) f d m=a \int_{X} f d m \text { for all } a \in C(S)
$$

and all $f \in L^{1}(X, m)$ then $\pi$ is unique and $\pi$ is an algebra homomorphism.

Suppose $\pi_{1}$ and $\pi_{2}$ are two such functions. For each $a \in C(S)$ and all $f \in L^{1}(X, m)$

$$
\int_{X}\left(\pi_{1}(a)-\pi_{2}(a)\right) f d m=a \int_{X} f d m-a \int_{X} f d m=0 .
$$

So, by Lemma $2.1, \pi_{1}(a)=\pi_{2}(a)$. Thus $\pi$ is unique. 
For each $a, b \in C(S)$ and all $f \in L^{1}(X, m)$

$$
\int_{X} \pi(a b) f d m=a b \int_{X} f d m=a \int_{X} \pi(b) f d m=\int_{X} \pi(a) \pi(b) f d m .
$$

Then, by Lemma $2.1, \pi(a b)=\pi(a) \pi(b)$. A similar argument shows that $\pi$ is linear.

We observe that if $a$ is a positive element of $C(S)$ then $\pi(a)=\pi\left(a^{1 / 2} a^{1 / 2}\right)=\pi\left(a^{1 / 2}\right)^{2}$ $\geqq 0$. So $\pi$ is a positive linear map and thus bounded with $\|\pi\|=\|\pi(1)\|=1$.

Definition 2.1. Let $m$ be a $C(S)$-valued measure on $(X, \mathscr{B})$. If there exists an algebra homomorphism $\pi: C(S) \rightarrow L^{\infty}(X, m)$ such that

$$
\int_{X} \pi(a) f d m=a \int_{X} f d m
$$

for all $a \in C(S)$ and all $f \in L^{1}(X, m)$ then $m$ is a modular measure (with respect to $\pi$ ).

It follows from Lemma 2.2 that $\pi$ is unique.

It is clear that when $m$ is modular with respect to $\pi$ then $m^{*}$ is also modular with respect to $\pi$.

When $m$ is a modular measure with respect to $\pi$ we write $a \cdot[f]$ for $\pi(a)[f]$. It is then apparent that $L^{p}(X)$ is a module over $C(S)$. Further, when $\phi: L^{1}(X) \rightarrow C(S)$ is defined by $\phi(f)=\int_{X} f d m, \phi$ is a module homomorphism of $L^{1}(X)$ into $C(S)$.

Proposition 2.1. Let $m$ be a $C(S)$-valued measure on $(X, \mathscr{B})$ which is modular with respect to $\pi$. Then there exists an idempotent $e \in C(S)$ such that

(i) $\pi(a)=0$ if and only if $a=e a$.

(ii) $\int_{X} f d m \in(1-e) C(S)$ for each $f \in L^{1}(X, m)$.

(iii) $\pi$ is an algebra isomorphism of $(1-e) C(S)$ into $L^{\infty}(X, m)$.

Let $\mathscr{F}=\left\{a \in C(S): 0 \leqq a \leqq 1\right.$ and $a \int_{X} f d m=0$ for all $\left.f \in L^{1}(X, m)\right\}$. Let $e=\bigvee \mathscr{F}$ then $1 \geqq e$ and so $e \geqq e^{2}$. If $b \in \mathscr{F}$ then $b^{1 / 2} \in \mathscr{F}$ and so $e^{2} \geqq b$. Thus $e^{2} \geqq e \geqq e^{2}$, that is, $e$ is an idempotent.

For each positive $f \in L^{1}(X, m), e \int_{X} f d m=\bigvee\left\{b \int_{X} f d m: b \in \mathscr{F}\right\}=0$, and so $e \in \mathscr{F}$. Since $\int_{X} \pi(e) f d m=e \int_{X} f d m=0$ for all $f \in L^{1}(X, m)$, it follows from Lemma 2.1 that $\pi(e)=0$.

Suppose $\pi(a)=0$. Because $\pi$ is a lattice homomorphism $\pi(|a|)=|\pi(a)|=0$. So

$$
|a| \int_{X} f d m=\int_{X} \pi(|a|) f d m=0
$$

for all $f \in L^{1}(X, m)$. Hence $|a| /(\|a\|+1) \in \mathscr{F}$ and thus $e|a|=|a|$. So $a=e a$.

Since $e$ is an element of $\mathscr{F}$ it follows that

$$
\int_{X} f d m \in(1-e) C(S) \text { for each } f \in L^{1}(X, m) .
$$

We observe that $\pi((1-e) a)=0$ if and only if $(1-e) a=e(1-e) a=0$. This completes the proof. 
Proposition 2.2. Let $X$ be a compact Hausdorff space and $\pi: C(S) \rightarrow C(X)$ an algebra homomorphism. Let $T: C(X) \rightarrow C(S)$ be a positive linear map such that $T(\pi(a) f)=a T f$ for all $a \in C(S)$ and $f \in C(X)$. Let $m$ be the unique quasi-regular $C(S)$-valued Borel measure on $X$ such that

$$
\int_{X} f d m=T f \text { for all } f \in C(X) .
$$

Let $N$ be the natural map of $C(X)$ into $L^{\infty}(X, m)$. Then $m$ is a modular measure with respect to $N \circ \pi$.

The existence, uniqueness and quasi-regularity of $m$ follows from Theorem 4.1 of [6].

Let $f^{*}$ be a positive bounded lower semicontinuous function on $X$ and let $a$ be a positive element of $C(S)$. Then

$$
\begin{aligned}
a \int_{X} f^{*} d m & =a\left[\bigvee\left\{\int_{X} g d m: 0 \leqq g \leqq f^{*} \text { and } g \in C(X)\right\}\right] \\
& =\bigvee\left\{a \int_{X} g d m: 0 \leqq g \leqq f^{*} \text { and } g \in C(X)\right\} \\
& =\bigvee\left\{a T g: 0 \leqq g \leqq f^{*} \text { and } g \in C(X)\right\} \\
& =\bigvee\left\{\int_{X} \pi(a) g d m: 0 \leqq g \leqq f^{*} \text { and } g \in C(X)\right\} \\
& =\int_{X} \pi(a) f^{*} d m
\end{aligned}
$$

since $\left\langle\pi(a) g: 0 \leqq g \leqq f^{*}\right.$ and $\left.g \in C(X), \leqq\right\rangle$ is an increasing net which converges pointwise to $\pi(a) f^{*}$.

Let $B^{\infty}(X)$ be the bounded Borel functions on $X$ and let $\mathscr{A}_{0}$ be the subalgebra of $B^{\infty}(X)$ consisting of all functions of the form $f^{*}-g^{*}$ where $f^{*}$ and $g^{*}$ are positive bounded lower semicontinuous functions.

Consider the collection of all algebras $\mathscr{A}$ such that (1) $\mathscr{A}_{0} \subset \mathscr{A} \subset B^{\infty}(X)$ and (2) $f \in \mathscr{A}$ and $a \in C(S)$ implies $a \int_{X} f d m=\int_{X} \pi(a) f d m$. Since $\mathscr{A}_{0}$ is in the collection, the collection is not empty. By Zorn's Lemma there is a maximal such algebra, $\mathscr{M}$, say. By appealing to the generalised Dominated Convergence Theorem proved in [6] we can show that the algebra of all functions which are pointwise limits of uniformly bounded sequences of functions in $\mathscr{M}$ is in the collection. It follows from the maximality of $\mathscr{M}$ that the pointwise limit of a uniformly bounded sequence of functions in $\mathscr{M}$ is in $\mathscr{M}$. Hence $\mathscr{M}=B^{\infty}(X)$. It now follows that $m$ is modular with respect to $N \circ \pi$.

This provides us with examples of modular measures. We shall now give a simple example of a measure which is not modular. In the introduction we defined measures $l$ and $m$ in the special case where $X=S=\{a, b\}$ a two-point space. It is easy to verify that these measures are both modular but we shall show that $l+2 m$ 
is not modular. Suppose $l+2 m$ is modular with respect to $\pi$. Let $x$ and $y$ be distinct real numbers and let $\pi(x, y)=(c, d)$. Let $f:\{a, b\} \rightarrow \boldsymbol{R}$ be defined by $f(a)=1$ and $f(b)=0$. Then, by the supposed modularity of $l+2 m$,

$$
\int_{\{a, b\}}(c, d) f d(l+2 m)=(x, y) \int_{\{a, b\}} f d(l+2 m) .
$$

So

$$
c f(a)(1,2)+d f(b)(2,1)=(x, y)[f(a)(1,2)+f(b)(2,1)] .
$$

Thus $(c, 2 c)=(x, 2 y)$ and so $x=y$ which is a contradiction. It follows that $l+m$ is not modular.

Let $e$ be an element of $C(S)$. We call $e$ a projection or idempotent when $e=e^{2}$, that is, when $e$ is the characteristic function of a clopen subset of $S$. Let $\left\{E_{j}: j \in J\right\}$ be a family of clopen subsets of $S$ and let $E$ be the closure of $\cup E_{j}$. Then $E$ is a clopen set and is the least upper bound of $\left\{E_{j}: j \in J\right\}$ in the Boolean algebra of clopen subsets of $S$. Further, $\chi_{E}$ is the least upper bound in $C(S)$ of the family $\left\{\chi_{E_{j}}: j \in J\right\}$.

We say $\left\{e_{j}: j \in J\right\}$ is a family of orthogonal projections in $C(S)$ when the functions $e_{j}$ are idempotents such that $e_{j} e_{k}=0$ when $j \neq k$, that is, they are characteristic functions of pairwise disjoint clopen subsets of $S$.

Proposition 2.3. Let $\left\{e_{j}: j \in J\right\}$ be a family of orthogonal projections in $C(S)$ and let $e=\bigvee\left\{e_{j}: j \in J\right\}$. Let $m$ be a modular measure with respect to $\pi$. Let $1 \leqq p<\infty$ and $f \in \mathscr{L}^{p}(X)$. If $e_{j} \cdot[f]=\pi\left(e_{j}\right)[f]=0$ for each $j \in J$ then $e \cdot[f]=0$.

We recall from the concluding remark of Lemma 2.2 that since $\pi$ is an algebra homomorphism it is a positive linear map.

We observe

$$
e_{j} \int_{X}|f|^{p} d m^{*}=\int_{X}\left|\pi\left(e_{j}\right) f\right|^{p} d m^{*}=0
$$

Thus

$$
e \int_{X}|f|^{p} d m^{*}=\bigvee_{j \in J} e_{j} \int_{X}|f|^{p} d m^{*}=0
$$

But

$$
\int_{X}|\pi(e) f|^{p} d m^{*}=\int_{X} \pi(e)|f|^{p} d m^{*}=e \int_{X}|f|^{p} d m^{*} .
$$

The result follows.

COMPLEX $L^{p}$-SPACES. We define $\mathscr{L}^{p}(X, m, C)$ to be the set of complex valued measurable functions $f$ on $X$ such that $|f| \in \mathscr{L}^{p}(X, m)$ and define the pseudonorm of $f$ to be the pseudonorm of $|f|$ as an element of $\mathscr{L}^{p}(X, m)$. It causes no clash with earlier notation if we denote the pseudonorm on $\mathscr{L}^{p}(X, m, C)$ by \|\|$_{p}$. Let $L^{p}(X)$ 
be the quotient of $\mathscr{L}^{p}(X, m, C)$ by the kernel of the pseudonorm \|\|$_{p}$. Each element of $L^{p}(X)$ is of the form $\xi+i \eta$ where $\xi$ and $\eta$ are elements of $L^{p}(X)$ and

$$
\max \left(\|\xi\|_{p},\|\eta\|_{p}\right) \leqq\|\xi+i \eta\|_{p} \leqq\|\xi\|_{p}+\|\eta\|_{p},
$$

so a sequence in $L^{p}(X)$ converges iff its real and imaginary parts converge. Thus $L^{p}(X)$ is a complex Banach space in which $L^{p}(X)$ is naturally embedded. Further, when $m$ is a modular measure, $L^{p}(X)$ is a module over $C(S)$, the ring of continuous complex-valued functions on $S$.

Let $\phi(f)=\phi(\mathscr{R}(f))+i \phi(\mathscr{I}(f))$ for $f \in L^{1}(X)$. Then $\phi$ is a module homomorphism of $L^{1}(X)$ into $C(S)$ when $m$ is a modular measure.

In [7] a pre-Kaplansky-Hilbert module over $C(S)$ is defined to be a module $K$ over $C(S)$ equipped with a $C(S)$-valued "inner product" such that for $x, y, z$ in $K$ and $a, b$ in $C(S)$

(1) $(x, y)=(y, x)^{*}$

(2) $(a x+b z, y)=a(x, y)+b(z, y)$

(3) $(x, x) \geqq 0$ with $(x, x)=0$ only if $x=0$.

LEMMA 2.3. Let $m$ be a modular measure. Then $L^{2}(X)$ is a pre-Kaplansky-Hilbert module over $C(S)$ when the inner product is defined by $(f, g)=\phi\left(f g^{*}\right)$.

3. Ample measures. A Kaplansky-Hilbert module over $C(S)$ is a pre-Kaplansky-Hilbert module over $\boldsymbol{C}(S)$ satisfying three completeness conditions, see [4].

On inspecting the definition of a Kaplansky-Hilbert module we see that when $m$ is a modular measure the pre-Kaplansky-Hilbert module $L^{2}(X)$ satisfies two of the completeness conditions.

(1) $L^{2}(X)$ is a Banach space with respect to the norm induced by the inner product.

(2) Let $\left\{e_{j}: j \in J\right\}$ be a family of orthogonal projections in $C(S)$ with

$$
e=\bigvee\left\{e_{j}: j \in J\right\}
$$

Then it follows from Lemma 2.1 that if $\xi \in L^{2}(X)$ and $e_{j} \cdot \xi=0$ for all $j \in J$ then $e \cdot \xi=0$.

So $L^{2}(X)$ will be a Kaplansky-Hilbert module iff it satisfies the third completeness condition i.e.

(3) Let $\left\{e_{j}: j \in J\right\}$ be a family of orthogonal projections in $C(S)$ with $\bigvee e_{j}=1$. Let $\left\{\xi_{j}: j \in J\right\}$ be a norm bounded family in $L^{2}(X)$. Then there exists $\xi \in L^{2}(X)$ such that $e_{j} \cdot \xi=e_{j} \cdot \xi_{j}$ for all $j \in J$.

Definition 3.1. A $C(S)$-valued measure $m$ is ample (with respect to $\pi$ ) when $m$ is modular (with respect to $\pi$ ) and $L^{2}(X)$ is a Kaplansky-Hilbert module.

It is clear that a modular measure $m$ is ample iff condition (3) above is satisfied.

Definition 3.2. Let $\mathscr{L}$ be any lattice. $\mathscr{L}$ satisfies the upper (lower) countable chain condition if, when $\mathscr{S}$ is a nonempty subset of $\mathscr{L}$ which is bounded above (below), then there is a countable set $\mathscr{S}_{0} \subset \mathscr{S}$ such that $\mathscr{S}_{0}$ and $\mathscr{S}$ have the same set 
of upper (lower) bounds. $\mathscr{L}$ satisfies the countable chain condition when $\mathscr{L}$ satisfies both the upper and lower countable chain conditions.

In the special case where $\mathscr{L}$ is a Boolean algebra this is equivalent to the condition that each set of pairwise disjoint elements of $\mathscr{L}$ be countable. This equivalence is proved by Halmos in $\$ 14$ of [2].

The following proposition gives a convenient sufficient condition for a measure to be ample.

Proposition 3.1. Let $m$ be a $C(S)$-valued modular measure. If the Boolean algebra of clopen subsets of $S$ satisfies the countable chain condition then $m$ is ample.

Let $\left\{e_{j}: j \in J\right\}$ be a family of orthogonal projections in $C(S)$ with $\bigvee\left\{e_{j}: j \in J\right\}$ $=1$. By hypothesis $J$ is countable and so may be assumed to be the set of natural numbers.

Let $\left\{f_{j}\right\} j=1,2, \ldots$ be a sequence in $\mathscr{L}^{2}(X)$ and suppose there exists a constant $M$ such that $\left\|f_{j}\right\|_{2} \leqq M$ for each $j$. Then

$$
\sum_{j=1}^{n} \phi\left(e_{j} \cdot f_{j}^{2}\right)^{1 / 2}=\sum_{j=1}^{n} e_{j} \phi\left(f_{j}^{2}\right)^{1 / 2} \leqq M \sum_{j=1}^{n} e_{j}
$$

Thus $\sum_{j=1}^{n} \phi\left(e_{j} \cdot f_{j}^{2}\right)^{1 / 2} \leqq M 1$. By Proposition 1.1 there exists $f \in \mathscr{L}^{2}(X)$ such that

Thus

$$
\operatorname{LIM}_{n} \phi\left(\left|f-\sum_{1}^{n} e_{j} \cdot f_{j}\right|^{2}\right)^{1 / 2}=0 .
$$

Thus

$$
\underset{n}{\operatorname{LIM}} e_{r} \phi\left(\left|f-\sum_{1}^{n} e_{j} \cdot f_{j}\right|^{2}\right)^{1 / 2}=0
$$

$$
\underset{n}{\operatorname{LIM}} \phi\left(\left|e_{r} \cdot f-e_{r} \cdot f_{r}\right|^{2}\right)^{1 / 2}=0
$$

So $e_{r} \cdot[f]=e_{r} \cdot\left[f_{r}\right]$.

It now follows that $L^{2}(X)$ satisfies all the completeness conditions necessary for it to be a Kaplansky-Hilbert module so $m$ is an ample measure.

Proposition 3.2. Let $S$ be extremally disconnected. The Boolean algebra of clopen subsets of $S$ satisfies the countable chain condition if and only if $C(S)$ satisfies the condition.

It is clear that if $C(S)$ satisfies the countable chain condition then so does the Boolean algebra of idempotents in $C(S)$.

Suppose the Boolean algebra of clopen subsets of $S$ satisfies the countable chain condition. Let $\left\{a_{j}: j \in J\right\}$ be a family of functions in $C(S)$ which is bounded above by a constant $M$. For each real number $r$ we define the following sets:

$$
O(j, r)=\left\{s \in S: a_{j}(s)>r\right\}, \quad K(j, r)=\operatorname{cl}(O(j, r)), \quad K(r)=\operatorname{cl}\left(\bigcup_{j \in J} O(j, r)\right) .
$$

Then $K(r)$ and $K(j, r)$ are clopen and $K(r)=\bigvee_{j \in J} K(j, r)$. 
Observe that $r_{1}<r_{2}$ implies $K\left(r_{1}\right) \supset K\left(r_{2}\right)$. If $r>M$ and $K(r) \neq \varnothing$ then $a_{j}(s)>M$ for some $j$ and $s$, which is false. So an upper bounded finite-valued function can be defined by

$$
a(s)=\sup \{\rho: \rho \text { is rational and } s \in K(\rho)\} .
$$

We show that $a$ is continuous and the least upper bound of $\left\{a_{j}: j \in J\right\}$.

If $r<a(s)$ then $s \in K(r)$. If $s \in K(t)$ then $a(s) \geqq t$ so $a(s)<t$ implies $s \notin K(t)$. For each open interval $(r, t)$,

$$
\{s: r<a(s)<t\}=\bigcup_{n=1}^{\infty}\left(K\left(r+\frac{1}{n}\right)-K\left(t-\frac{1}{n}\right)\right)
$$

and so $a$ is continuous.

If $a_{j}(s)>\rho$ where $\rho$ is rational then $s \in K(\rho)$ and so $a(s) \geqq \rho$. Thus $a$ is an upper bound for $\left\{a_{j}: j \in J\right\}$. Let $b$ be another upper bound and suppose $a\left(s_{0}\right)>b\left(s_{0}\right)$ for some $s_{0} \in S$. Let $\rho$ be a rational strictly between $a\left(s_{0}\right)$ and $b\left(s_{0}\right)$. Then $s_{0} \in K(\rho)$. Let $U$ be the open set $\{s \in S: a(s)>\rho>b(s)\}$. We have $a_{j}(s) \leqq b(s)<\rho$ for each $s \in U$ and so $U \cap O(j, \rho)=\varnothing$ for each $j \in J$. Thus $s_{0}$ is not in the closure of $\bigcup_{j \in J} O(j, \rho)$ that is, $s_{0} \notin K(\rho)$. This contradiction shows that $a$ is the least upper bound.

Let $\rho$ be rational. Because the Boolean algebra of clopen subsets of $S$ satisfies the countable chain condition we can choose a countable set $J(\rho) \subset J$ such that

$$
\bigvee\{K(j, \rho): j \in J(\rho)\}=\bigvee\{K(j, \rho): j \in J\}
$$

Let $I=\bigcup\{J(\rho): \rho$ is rational $\}$ then $I$ is countable and $K(\rho)=\bigvee\{K(j, \rho): j \in I\}$ for each rational $\rho$. It follows, by repeating the construction in the first part of the proof, that $a$ is the least upper bound of $\left\{a_{j}: j \in I\right\}$ which is a countable subset of $\left\{a_{j}: j \in J\right\}$. This establishes the result.

When $C(S)$ is isomorphic to a commutative von Neumann algebra over a separable Hilbert space or, equivalently, when $C(S) \cong L^{\infty}(X, \mu)$ where $\mu$ is a finite real valued measure, then $C(S)$ satisfies the countable chain condition.

Let $B^{\infty}[0,1]$ be the bounded Borel measurable functions on $[0,1]$ and let $\mathscr{M}$ be the functions in $B^{\infty}[0,1]$ which vanish outside a meagre set. Then $B^{\infty}[0,1] / \mathscr{M}$ is isomorphic to $C(S)$ where $S$ is the Boolean space of the Boolean algebra of regular open subsets of $[0,1]$. This Stone algebra satisfies the countable chain condition and is not isomorphic to any von Neumann algebra.

PROPOSITION 3.3. Let $m$ be a $C(S)$-valued measure which is not required to be modular. If $C(S)$ satisfies the countable chain condition then so also does $L^{1}(X)$. Further, if $L^{1}(X)$ satisfies the countable chain condition and $m$ is a finite measure then $L^{\infty}(X)$ is a Stone algebra satisfying the countable chain condition.

Let $\left(\xi_{j}: j \in J, \leqq\right)$ be an increasing net in $L^{1}(X)$ which is bounded above by an element of $L^{1}(X)$. Then $\left(\phi\left(\xi_{j}\right): j \in J, \leqq\right)$ is an increasing net in $C(S)$ which has a least upper bound $b$. Because $C(S)$ satisfies the countable chain condition we can find a countable set $I \subset J$ such that $b$ is the least upper bound of $\left\{\phi\left(\xi_{j}\right): j \in I\right\}$. 
Enumerate $I,\{i(1), i(2), \ldots\}$ and define, inductively, a countable subset $\{j(1)$, $j(2), \ldots\}$ of $J$ such that $\xi_{j(r)} \geqq \xi_{i(r)}$ for each $r$ and $\left\{\xi_{j(r)}\right\} r=1,2, \ldots$ is a monotonic sequence. Then $\bigvee_{r=1}^{\infty} \phi\left(\xi_{j(r)}\right)=b$. By the Monotone Convergence Theorem, Proposition 3.3 of [6], there exists $[f] \in L^{1}(X)$ such that if $f_{j} \in \xi_{j}$ for each $j$ then $\left\{f_{j(r)}\right\}$ $r=1,2, \ldots$ converges to $f$ pointwise almost everywhere as $r \rightarrow \infty$ and $\phi(f)=b$.

Now we must show that $[f]$ is the least upper bound of the $\xi_{j}$. Clearly no smaller element of $L^{1}(X)$ will do. Let $n \in J$. Then define inductively, as above, an increasing sequence $\left\{\xi_{k(r)}\right\} r=1,2, \ldots$ such that

$$
\xi_{k(r)} \geqq \xi_{n} \vee \xi_{j(r)} \text { for } r=1,2, \ldots
$$

Then there is a $[g] \geqq \xi_{n}$ in $L^{1}(X)$ such that

$$
\phi(g)=\bigvee_{r=1}^{\infty} \phi\left(\xi_{k(r)}\right)=b
$$

and $[g] \geqq[f]$. Since $\phi(g-f)=b-b=0$ this implies $[f]=[g] \geqq \xi_{n}$.

It follows from this result and the fact that $L^{1}(X)$ is a lattice that each nonempty subset of $L^{1}(X)$ which is bounded above has a countable subset with the same least upper bound.

Suppose $L^{1}(X)$ satisfies the countable chain condition and $m$ is a finite measure. Then $L^{\infty}(X) \subset L^{1}(X)$ and so $L^{\infty}(X)$ satisfies the countable chain condition. Since $L^{\infty}(X) \cong C(E)$, where $E$ is a compact Hausdorff space, and, since $L^{\infty}(X)$ is boundedly $\sigma$-complete, $L^{\infty}(X)$ is a Stone algebra. This completes the proof.

We have shown that a sufficient condition for a modular measure to be ample is that it takes its values in a Stone algebra satisfying the countable chain condition. This condition is simple and convenient for applications but is not necessary. We give a further sufficient condition for a measure to be ample. Since no use is made of this result we suppress the rather tedious proof.

Definition 3.3. $C(S)$ satisfies the local countable chain condition if there is a family of pairwise disjoint clopen sets $\left\{E_{j}: j \in J\right\}$ such that

(1) $C\left(E_{j}\right)$ satisfies the countable chain condition.

(2) $\bigvee\left\{E_{j}: j \in J\right\}=S$.

Kaplansky shows in $\$ 10$ of [3] that any von Neumann algebra satisfies the local countable chain condition.

Proposition 3.4. Let $X, S, \pi$ and $T$ satisfy the hypotheses of Proposition 2.2 and suppose that $C(S)$ satisfies the local countable chain condition. Let $m$ be the measure constructed in Proposition 2.2. Then there is a $\sigma$-algebra $\mathscr{B}$ which contains the Borel sets and a measure $l$ on $\mathscr{B}$ such that $l E=m E$ for each Borel set $E$ and $l$ is ample.

If $C(S)$ is isomorphic to a von Neumann algebra in Proposition 3.4 then $m$ is regular. The regularity of $m$ can be used to show that each element of $\mathscr{B}$ is of the form $B \cup N$ where $B$ is a Borel set and $N \subset E$ where $E$ is a Borel set and $m E=0$. It follows that $L^{2}(X, m)=L^{2}(X, l)$ and so $m$ is ample. 
4. Absolute continuity and the generalized Radon-Nikodym Theorem. In this section $l$ and $m$ are modular $C(S)$-valued measures on a measurable space $(X, \mathscr{B})$. They are finite measures, that is, $l X \in C(S)$ and $m X \in C(S)$. When $f$ is any real valued $\mathscr{B}$-measurable function let $[f]_{l}\left([f]_{m}\right)$ be the set of $\mathscr{B}$-measurable functions differing from $f$ only on sets of zero $l$-measure ( $m$-measure).

Suppose $E \in \mathscr{B}$ and $m E=0$ implies $l E=0$. Then $[f]_{m} \subset[f]_{l}$ and so a natural map $N: L^{\infty}(X, m) \rightarrow L^{\infty}(X, l)$ can be defined by $N[f]_{m}=[f]_{l} . N$ is an algebra homomorphism onto $L^{\infty}(X, l)$.

DEFINITION 4.1. $l$ is absolutely continuous with respect to $m$ (modulo $\pi$ ) when

(1) If $E \in \mathscr{B}$ and $m E=0$ then $l E=0$.

(2) The map $\pi: C(S) \rightarrow L^{\infty}(X, m)$ is an algebra homomorphism such that $m$ is modular with respect to $\pi$ and $l$ is modular with respect to $N \circ \pi$.

LEMMA 4.1. Let l be absolutely continuous with respect to $m$ (modulo $\pi$ ) then $l+m$ is modular with respect to $\pi$.

We have $(l+m) E=0$ iff $l E=0$ and $m E=0$ and thus $(l+m) E=0$ iff $m E=0$. So $[g]_{m}=[g]_{l+m}$ whenever $g$ is measurable. Thus $L^{\infty}(X, m)=L^{\infty}(X, l+m)$. Also $\int_{X} g d(l+m)$ exists iff $\int_{X} g d l$ and $\int_{X} g d m$ exist. So $\mathscr{L}^{p}(X, l+m)=\mathscr{L}^{p}(X, l)$ $\cap \mathscr{L}^{p}(X, m)$ for $1 \leqq p<\infty$.

Let $a \in C(S)$ and suppose $\pi(a)=[g]_{m}=[g]_{l+m}$ where $g$ is bounded. Let $h \in$ $\mathscr{L}^{1}(X, l+m)=\mathscr{L}^{1}(X, l) \cap \mathscr{L}^{1}(X, m)$. Then

$$
\begin{aligned}
\int_{X} \pi(a) h d(l+m) & =\int_{X} g h d l+\int_{X} g h d m \\
& =\int_{X} N \circ \pi(a) h d l+\int_{X} \pi(a) h d m \\
& =a \int_{X} h d l+a \int_{X} h d m \\
& =a \int_{X} h d(l+m) .
\end{aligned}
$$

Definition 4.2. $l$ is amply absolutely continuous with respect to $m$ (modulo $\pi$ ) when $l$ is absolutely continuous with respect to $m$ (modulo $\pi$ ) and the $\pi$-modular measure $l+m$ is ample.

We note that if $C(S)$ satisfies the countable chain condition and $l+m$ is a modular measure then $l+m$ is ample. So when $C(S)$ satisfies the countable chain condition Definition 4.2 is superfluous.

LEMMA 4.2. Let $q$ be an ample modular measure and let $H: L^{2}(X) \rightarrow C(S)$ be a module homomorphism which is continuous when $L^{2}(X)$ and $C(S)$ are given their norm topologies. Then there is a unique $\xi \in L^{2}(X)$ such that

$$
H f=\int_{X} f \xi d q \text { for all } f \in L^{2}(X)
$$


Each element of $L^{2}(X)$ can be expressed uniquely in the form $f+i g$ where $f$ and $g$ are in $L^{2}(X)$. So $\boldsymbol{h}: \boldsymbol{L}^{2}(X) \rightarrow \boldsymbol{C}(S)$ can be defined by $\boldsymbol{h}(f+i g)=H f+i H g$. Then $\boldsymbol{h}$ is a module homomorphism of $L^{2}(X)$, regarded as a module over $C(S)$, into $C(S)$. We have

$$
\begin{aligned}
\|h(f+i g)\| & \leqq\|H f\|+\|H g\| \\
& \leqq\|H\|\left\{\left\|\left(\int_{X} f^{2} d q\right)^{1 / 2}\right\|+\left\|\left(\int_{X} g^{2} d q\right)^{1 / 2}\right\|\right\} \\
& \leqq 2\|H\|\left\|\left(\int_{X}\left(f^{2}+g^{2}\right) d q\right)^{1 / 2}\right\| .
\end{aligned}
$$

So $h$ is a bounded operator.

Since $L^{2}(X)$ is a Kaplansky-Hilbert module it now follows from Theorem 5 of [4] that there is a unique $\zeta=\xi+i \eta$ such that

$$
\boldsymbol{h}(z)=\int_{X} z \zeta^{*} d q \text { for all } z \in L^{2}(X)
$$

Thus

$$
H f=\int_{X} f \xi d q-i \int_{X} f \eta d q \text { for all } f \in L^{2}(X)
$$

Since $H$ is $C(S)$-valued the result follows.

We now come to the main result.

THEOREM 4.1. Let $l$ and $m$ be finite $C(S)$-valued measures on the measurable space $(X, \mathscr{B})$. Let l be amply absolutely continuous with respect to $m$. Then there is a positive $\mathscr{B}$-measurable function $f$ such that

$$
l E=\int_{E} f d m \text { for each } E \in \mathscr{B} .
$$

Let $\pi: C(S) \rightarrow L^{\infty}(X, m)$ be such that $m$ is $\pi$-modular and $l$ is $N \circ \pi$-modular, where $N$ is the natural map of $L^{\infty}(X, m)$ onto $L^{\infty}(X, l)$. By Lemma $4.1, l+m$ is $\pi$-modular. Also $\left[g_{m}\right]=[g]_{l+m}$ for each $\mathscr{B}$-measurable $g$ and $\mathscr{L}^{2}(X, l+m)=$ $\mathscr{L}^{2}(X, l) \cap \mathscr{L}^{2}(X, m)$.

Let $[g]_{l+m} \in L^{2}(X, l+m)$ then $g \in \mathscr{L}^{2}(X, m)$. Appealing to Lemma 1.5 we have that $\int_{X} g d m$ exists and

$$
\left|\int_{X} g d m\right| \leqq\left(\int_{X} g^{2} d m\right)^{1 / 2}(m X)^{1 / 2}
$$

So we can define $H: L^{2}(X, l+m) \rightarrow C(S)$ by $H[g]_{l+m}=\int_{X} g d m$. Since $l+m$ and $m$ are modular with respect to $\pi, H$ is a module homomorphism. Further

$$
\left\|H[g]_{l+m}\right\| \leqq\left\|(m X)^{1 / 2}\right\|\left\|\left(\int_{X} g^{2} d(l+m)\right)^{1 / 2}\right\| .
$$

Thus $H$ is a bounded linear operator. 
By Lemma 4.2 there exists $h \in \mathscr{L}^{2}(X, l+m)$ such that

$$
H[g]_{l+m}=\int_{X} g h d(l+m) .
$$

Let $E_{0}=\{x \in X: h(x) \leqq 0\}$ and

$$
E_{n}=\{x \in X: h(x) \geqq 1+1 / n\} \text { for } n=1,2, \ldots
$$

Then

$$
m E_{0}=\int_{E_{0}} h d(l+m) \leqq 0
$$

and

$$
m E_{n}=\int_{E_{n}} h d(l+m) \geqq\left(1+\frac{1}{n}\right)\left(l E_{n}+m E_{n}\right)
$$

so $0 \geqq\left(1+n^{-1}\right) l E_{n}+n^{-1} m E_{n}$.

It follows that we may assume without loss of generality that $0<h(x) \leqq 1$ for all $x \in X$. Then $1 / h$ is a well defined measurable function.

Let $E \in \mathscr{B}$. Fix an arbitrary natural number $n$ and let

$$
F_{r}=\{x \in E: n /(r+1)<h(x) \leqq n / r\}
$$

for each natural number $r$. For all $x \in F_{r}$ we have $r / n \leqq 1 / h(x)<(r+1) / n$. So

$$
\frac{r}{n} m F_{r} \leqq \int_{F_{r}} \frac{1}{h} d m \leqq \frac{r+1}{n} m F_{r}
$$

Also

$$
\frac{n}{r+1}(l+m) F_{r} \leqq \int_{F_{r}} h d(l+m)=m F_{r} \leqq \frac{n}{r}(l+m) F_{r} .
$$

Thus

$$
r m F_{r} / n \leqq(l+m) F_{r} \leqq(r+1) m F_{r} / n .
$$

From (1) and (2) we have

$$
-\frac{1}{n} m F_{r} \leqq \int_{X} \chi_{F_{r}} \frac{1}{h} d m-(l+m) F_{r} \leqq \frac{1}{n} m F_{r} .
$$

By this inequality and Proposition 3.3 of [6] we find

$$
\int_{E} \frac{1}{h} d m \text { exists and }\left\|(l+m) E-\int_{E} \frac{1}{h} d m\right\| \leqq \frac{1}{n}\|m X\| \text {. }
$$

Since $n$ is arbitrary this gives $l E=\int_{E}(1 / h-1) d m$.

Using Theorem 4.1 we obtain an analogue of the Hahn Decomposition Theorem. In the following lemmas and theorem $X$ is a compact Hausdorff space and $C(X)$ the ring of continuous real valued functions on $X$, and $B_{0}^{\infty}(X)$ the ring of bounded Baire functions on $X$. 
Lemma 4.3. Let $T: C(X) \rightarrow C(S)$ be a bounded linear operator. Then there exist positive linear operators $T^{+}$and $T^{-}$such that $T=T^{+}-T^{-}$and if $T=P-Q$ where $P$ and $Q$ are positive operators then $P \geqq T^{+}$and $Q \geqq T^{-}$. Further, if $\pi: C(S) \rightarrow C(X)$ is an algebra homomorphism such that $T(\pi(a) f)=$ aTf for each $a \in C(S)$ and $f \in C(X)$ then $T^{+}(\pi(a) f)=a T^{+} f$ and $T^{-}(\pi(a) f)=a T^{-} f$ for each $a \in C(S)$ and $f \in C(X)$.

For each positive $f \in C(X)$ the set $\{T(h): 0 \leqq h \leqq f$ and $h \in C(X)\}$ is a subset of $C(S)$ which is bounded above and so has a least upper bound and so we define

$$
T^{+} f=\bigvee\{T h: 0 \leqq h \leqq f \text { and } h \in C(X)\}
$$

Then $T^{+}$is additive on the positive cone of $C(X)$ and, when $\pi$ exists, $T^{+}(\pi(a) f)$ $=a T^{+} f$ for each positive $a \in C(S)$.

For an arbitrary element $g$ of $C(X)$ we define

$$
T^{+} g=T^{+}(g+\|g\|)-\|g\| T^{+} 1 .
$$

Let $T^{-}=T^{+}-T$. Then $T^{+}$and $T^{-}$have the required properties.

LEMMA 4.4. Let $m$ be a $C(S)$-valued Baire measure on the compact Hausdorff space $X$. Suppose $h$ is a bounded Baire measurable function on $X$ such that $\int_{X} h g d m$ $\geqq 0$ whenever $g$ is a positive element of $C(X)$. Then $h \geqq 0$ except on a set of zero m-measure.

Let $\phi(g)=\int_{X} h g d m$ for each $g \in C(X)$. Then $\phi$ is a positive linear $C(S)$-valued map. So by Theorem 4.1 of [6] there is a unique $C(S)$-valued Baire measure $q$ such that $\phi(g)=\int_{X} g d q$ for each $g \in C(X)$.

Let $B_{0}^{\infty}(X)$ be the set of bounded Baire measurable functions on $X$ and let

$$
\mathscr{V}=\left\{f \in B_{0}^{\infty}(X): \int_{X} f d q=\int_{X} h f d m\right\} .
$$

Then $C(X) \subset \mathscr{V}$. Also the pointwise limit of a uniformly bounded sequence of functions in $\mathscr{V}$ is a function in $\mathscr{V}$.

Consider the collection of all algebras $\mathscr{A}$ such that $C(X) \subset \mathscr{A} \subset \mathscr{V}$. Order this family by set inclusion. It follows by a Zorn's Lemma argument that there is a maximal algebra $\mathscr{M}$. The algebra $\overline{\mathscr{M}}$ whose elements are the pointwise limits of uniformly bounded sequences of functions in $\mathscr{M}$ is in the collection and contains $\mathscr{M}$. Since $\mathscr{M}$ is maximal we have $\mathscr{M}=\overline{\mathscr{M}}$. Hence $\mathscr{M}=B_{0}^{\infty}(X)$ and so $\mathscr{V}=B_{0}^{\infty}(X)$.

Let $D_{n}=\{x: h(x) \leqq-1 / n\}$ then

$$
\int_{D_{n}} h d m=\int_{D_{n}} d q=q D_{n} \geqq 0 .
$$

So $-m D_{n} / n \geqq 0$ and thus $m \bigcup_{n=1}^{\infty} D_{n}=0$. The result follows.

THEOREM 4.2. Suppose $C(S)$ satisfies the countable chain condition or is isomorphic to a von Neumann algebra. Let $\pi: C(S) \rightarrow C(X)$ be an algebra homomorphism. Let $T: C(X) \rightarrow C(S)$ be a bounded linear map such that, for all $a \in C(S)$ and 
$f \in C(X), T \pi(a) f=a T f$. Let $m^{+}$and $m^{-}$be the unique $C(S)$-valued Baire measures on $X$ corresponding to $T^{+}$and $T^{-}$. Then there is a Baire set $A$ such that

$$
m^{+} E=m^{+} E \cap A \text { and } m^{-} E=m^{-} E \cap(X-A)
$$

for each Baire set $E$.

Let $|m|=m^{+}+m^{-}$. Then it follows from Theorem 4.1 that there is a positive Baire measurable function $g$ such that

$$
\int_{X} f d m^{+}=\int_{X} f g d|m| \text { for all } f \in B_{0}^{\infty}(X)
$$

So

$$
\int_{X} f d m^{-}=\int_{X} f(1-g) d|m| \text { for all } f \in B_{0}^{\infty}(X)
$$

We can suppose without loss of generality that $1-g$ is positive. So $0 \leqq g \leqq 1$.

We have $T f=\int_{X} f(2 g-1) d|m|$ for $f \in C(X)$. Let $A=\left\{x \in X: g(x) \geqq \frac{1}{2}\right\}$. Then

$$
T f=\int_{A} f|2 g-1| d|m|-\int_{X-A} f|2 g-1| d|m| .
$$

It follows from Lemma 4.3 that when $f$ is a positive element of $C(X)$,

$$
T^{+} f \leqq \int_{A} f|2 g-1| d|m|
$$

So

$$
\int_{X}\left\{(2 g-1) \chi_{A}-g\right\} f d|m| \geqq 0 \text { for all positive } f \text { in } C(X)
$$

Appealing to Lemma 4.4 we have

$$
\chi_{A} g-\chi_{\mathrm{X}-{ }_{A}} g-\chi_{A} \geqq 0
$$

except on a set of zero $|m|$-measure. Multiplying both sides of (1) by $\chi_{A}$ and recalling that $g \leqq 1$ we have $\chi_{A} g=\chi_{A}$ almost everywhere. Multiplying both sides of (1) by $\chi_{X-A}$ and recalling that $0 \leqq g$ we have $\chi_{X-A} g=0$ almost everywhere. Thus $g=g\left(\chi_{A}+\chi_{X-A}\right)=\chi_{A}$ except on a set of zero $|m|$-measure.

The theorem follows.

I wish to thank Dr. D. A. Edwards for suggesting the problem considered in this paper and for his helpful advice and criticism.

\section{REFERENCES}

1. E. E. Floyd, Boolean algebras with pathological order properties, Pacific J. Math. 5 (1955), 687-689.

2. P. R. Halmos, Boolean algebras, Van Nostrand, Princeton, N. J., 1963. 
3. I. Kaplansky, Algebras of type I, Ann. of Math. 56 (1952), 460-472.

4. - Modules over operator algebras, Amer. J. Math. 75 (1953), 839-858.

5. M. H. Stone, Boundedness properties in function lattices, Canad. J. Math. 1 (1949), 176-186.

6. J. D. Maitland Wright, Stone algebra valued measures and integrals, Proc. London Math. Soc. (to appear).

7. - , A spectral theorem for normal operators on a Kaplansky-Hilbert module, Proc. London Math. Soc. (to appear).

\section{Christ Church,}

OXFORD, ENGLAND 